\title{
Geriatric Consultation Team in Emergency Department: A business Case Report Analysis
}

\author{
Hanadi Al Hamadd ${ }^{1,2}$ and Navas Nadukkandiyil ${ }^{* 2,3}$ \\ ${ }^{1}$ Chairperson Geriatrics, Rumailah Hospital,Hamad Medical Corporation,Doha,Qatar \\ ${ }^{2}$ Weill Cornell Medical College, Qatar \\ ${ }^{3}$ Specialist \&Clinical Fellow, Rumailah Hospital, Qatar
}

Submission: March 22, 2017; Published: April 06, 2017

*Corresponding author: Navas Nadukkandiyil, Department of Geriatrics, Al-Rumailah hospital, Hamad Medical Corporation, p.o box 3050, Doha, Qatar, Tel: +97433050426; Fax: +97444398111; Email: drnavasnk30@gmail.com

Abstract

The proportion of older patients presenting to emergency departments (EDs) are increasing. ED are busy environments in which complex, older persons living in the community aged care facilities are sometimes subjected to emergency department lengths of stay more than expectation, excessive tests and complications. One possible solution, a geriatric physician-championed, emergency department with gerontological intervention team, which provides frontload assessment, early discharge planning. The aim of this Geriatric Emergency Department Intervention is to maximize the quality of care for this vulnerable elderly patients in a cost effective manner. The author would like to give a critical insight into the present system of treatment in the ED for elderly with a focus on quality improvements and how a change in the approach by the implementation of a Geriatric Consultation Team (GCT) in the ED would bring about a safety and quality improvement in the treatment of elderly.

\section{Introduction}

The elderly visit in the emergency services is at a higher rate with multiple reasons and results in higher rates of adverse health outcomes after disposition. This may be due to frail condition of elderly and further the injuries in the elderly often require more intensive therapy and greater resources with a different level of care as compared to similar injuries in young patients [1]. Several systematic reviews have examined the impact of interventions targeted at improving outcomes for the elderly visiting the emergency department(ED) with mixed results [2]. The nature of these interventions have ranged from comprehensive geriatric assessments (CGA) with specially trained ED teams and followup of elderly discharged from the ED. Desired outcomes have been variable which have included improvement in the quality of life, slower progression of functional decline and a reduction in number of ED visits. The writer believes that innovations to expand the traditional role of the emergency care system beyond the mere clinical treatment of the acute illness to fully addressing and facilitating the biomedical and psychosocial factors surrounding the acute illness of older patients as the way ahead. Addressing these factors early while patients are in or even en route to the Emergency Department will likely optimize emergency care to be more patients centered, efficient and costeffective [3].
The author would like to give a critical insight into the present system of treatment in the ED for elderly with a focus on quality improvements and how a change in the approach by the implementation of a Geriatric Consultation Team (GCT) in the ED would bring about a safety and quality improvement in the treatment of elderly. One randomized Australian study showed that senior's admission rates at 30 days could be reduced through assessment by a comprehensive geriatric assessment team (CGA) prior to their initial ED discharge [4]. The routine model [5] for ED care in the author's hospital consists of rapid patient evaluation, early diagnosis, treatment, and then disposition to home or admission to an inpatient unit as necessary. Such care processes focus only the needs of the health care system rather than the special care of the elderly. The elder patients may require initial multidisciplinary evaluation by a multidisciplinary geriatric consultation team for "geriatricizing" the present emergency department to a geriatric friendly emergency consultation model of care. This includes interdisciplinary staff education in evidence-based protocols and appropriate structural modifications to the physical space and these interventions have shown to successfully improve the quality of care by reducing length of stay and safety of older adults while lowering inpatient costs [6]. 


\section{Analyzing context on quality perspective}

Every staff in the hospital shares the common idea of delivering the safest, most effective and compassionate care to our patients. Many qualities based campaigns are in place to help to achieve the same by committing every team member in the improvement of quality and patient safety. This quality improvements have been designed in partnership with the Institute of Healthcare Improvement (IHI) who happens to be the world leaders in the science and practice of healthcare improvement. The team is working closely with the IHI throughout the campaign in learning and applying their wealth of knowledge and experience in quality and safety. Best Care Always' is one of the systematic, coordinated and focused approaches to institutional quality improvement. This campaign is designed to identify and implement consistent and sustainable best practice that will be at the fore front in delivering patient care.

There are two aspects to the initial stages of the quality design; one is the essential early work of identifying, inspiring, training and supporting individual champions of improvement; and the other is by measuring and analyzing systems to identify improvement $\mathrm{s}$ that can be applied across the institution. The team has been encouraged to think about systems analysis and to base its decision on sound evidence. The team will have to work to create an environment in which improvement is a part of everyone's life and work, whatever their role and set challenges to be the best at getting better. The team is focusing to create a quality based elder friendly approach in the ED as more elderly patients occupy ED beds per day with great difficulty in disposition. Hence creating a Geriatric Consultation Team (GCT) in the ED will help in reduction of length- of- stay of elderly patients across the hospital. In addition, it will alleviate the bed crises and increase the quality of care for elderly in the ED. This quality project recommends a modern, state-of-the- art Geriatric consultation team in ED with an eye for patient centered care.

\section{Rationale of GCT}

The elderly patients tend to have long-term and often complex needs and also tend to have more frequent interactions with the health system. They happen to be the most affected by the current lack of alignment in goals, incentives, and coordinated care delivery for elderly in the ED. They would also benefit from a better clinical integration; sharper focus on prevention; disease management and self-care; and tighter adherence to the best practices. This population constitutes a substantial and rapidly growing proportion of healthcare expenditure across the world population [7].

A great percentage of the elderly patients stay for a long time in emergency in many place, even after their acute medical problem is over. The reasons behind this are mainly social issues relating to the family not being able to take care of them at home. This is usually either due to lack of knowledge from the ED perspective of how to deal with these important issues. It is interesting to note that in this brief analysis of health care system in ED resulted in the realization of a lack of quality and safety based triaging for elderly patients as priority. So reforming the ED design by implementing a multidisciplinary geriatric consultation team(GCT)in emergency care beyond merely the clinical treatment of the acute illness to comprehensive care with importance to the physical and psychosocial factors surrounding the acute illness of older patients. Addressing these factors early while patients are on or even en route to the "front line" will likely optimize emergency care to be more patients centered, effective, efficient, and cost-effective.

\section{Frameworks and Model of Care}

The below analysis with clinical input from ED may help to identify the opportunity and type of intervention that may benefit the >65years population. It represents the system demands in institution for specialized care by geriatric ED multidisciplinary team. Hence a reshaping of system is required to change from a supply led system to a demand driven system as follows [8]. GCT will assess older adults (65+) and current community clients presenting to primary site EDs and will work in collaboration with the ED team and with the patient/family. GCT also will do a complete comprehensive assessment to determine the plan of care. GCT nurse and/or geriatric physician will review recommendations with ED physician/team that will be responsible for final discharge decision. GCT follow up calls to ensure follow up regarding transition of care, coordination of care, and to address any additional care needs, safety concerns, reinforce education and provide psychosocial support.

I. The API (Associate in process Improvement) PDSA model: Model of frame work performance implementation will provide improvement in the current system in a step wise manner.

II. Plan (Aim): To establish a Multi-Disciplinary Geriatric consultation team (GCT) in the ED

III. Do: To ensure elderly patients receive the right care in the right place at the right time.

IV. Study: By implementing above aim in ED, helps to reduce length-of-stay of elderly patients across the hospital; this team can also reduce number of readmissions to the hospitals and provide appropriate services for the elderly patients to regain their independence.

V. Act: It results in maximum functional independence of targeted people by assisting them in their activities of daily living (ADL) and instrumental activities of daily living (IADL) and re- integrate elderly patients back into their communities. The following changes in patients will result in an improvement such as preserve patients' independence, Improve quality of life of elderly patients, Assist in giving patients the feeling of usefulness. 


\section{Specific, measurable expected benefits}

GCT provides an alternative to hospital admission and enhances the emergency experience for older adults and existing home care sick patients which possibly avoids admission and future visits to the ED. Reducing avoidable hospital visits, readmissions within 72 hours, improves health outcomes and the health care experience while maintaining or reducing the cost of providing appropriate care. The ability to care for elderly people in their home effectively prevents risks associated with an ED visit and hospital admission and supports several key deliverables within national continuing care strategy. GCT teams provides comprehensive geriatric assessments (CGA) and creates care plans to optimize function and independence and help the elderly to be at home. Other Benefits [9].

i. Advocate for older adults and their families

ii. Educate and build elder friendly capacity within the ED, hospital and community

iii. Marketing/branding

iv. Facilitate transfer of patients to long term care unit

v. Easy transition from hospital to community through Home Health Care

vi. Reduce overall cost

vii. Risks associated with Change Model. (SWOT analysis)

I. Strengths: Include the comprehensive early assessment of elderly patients with an eye for early discharge. The involvement of a multidisciplinary team to assess the needs of the patients and plan to have a proper plan by the experts on the needs of the elderly. Improvements in quality and patient safety by a team trained in geriatrics with a focus on improvements and proactive planning.

II. Weakness: The inability of the present team to provide 24 hours care as this is only a pilot project. The inability to foresee the possible complications in the ED during the implementation of this model as it happens to be the first of this kind in whole of the country. Excessive reliance of the GCT by the ED physicians on trivial matters in treatment as there will be not be a clear demarcation of services during the early implementation phase.

III. Opportunity: Opportunity for improvement for individual members of the team work towards a common goal. Interactions with the ED team could give valuable inputs into the prevalence and distribution of diseases among the elderly. Opportunity to educate and train the ED team on the special needs of the elderly and possible interactions for quality improvement methods. Possibility of creating a clinical guideline for standardized care for the elderly in the ED with inputs from the ED team with the GCT as the clinical lead.
IV. Threats: Threat of burning out of clinical team due to excessive demand in a very busy ED. Lack of same goals for the two teams in place can lead to differences in opinions on further management. Involvement of a new team along with the number of consulting teams already present in the ED could lead to overcrowding in the ED. Many of the geriatric protocols and models of care [10]. That currently existence require research and validation in the emergency setting.

\section{The cost Analysis}

Considering financial standpoint, avoiding the costs of hospitalization for older adults is crucial for individuals and insurers alike. The author assumes that this project will also be of interest to EDs throughout the stakeholders. This project concludes with high level plan checklist of specific actions to monitor quality and safety improvements and focus on the accountability of the outcomes that matter [11].

\section{High-level plan:}

Geriatric assessment by GCT is geared toward decision making for rapid disposition to home or admission to acute geriatrics unit from ED. Thus, the service functions as a gatekeeper for admission to the acute care for elderly services, as well as coordinating geriatric follow-up both in-hospital (for patients admitted to other services) and in the community (for patients discharged home), via the outpatient geriatric clinic, home visits, or linkage to other community resources [12].

I. Create a well-planned team by 6 months:

i. Geriatric Physician,

ii. Geriatric clinical nurse,

iii. Home health care nurse

iv. Occupational therapist, Physical therapist and Speech therapist,

v. Case manager

vi. Clinical pharmacist,

vii. Dietician,

viii. Social worker. Consultations are received by referrals from primarily ED physicians to encompass all aspects of medical and psychosocial issues.

Role of the Geriatric Consultation Team Comprehensive geriatric assessments including, Fall risk, Memory, Frailty, Nutritional, Swallowing assessments. Community care coordination with the Mobile physician, transfer of care to Medicine/Acute Geriatric team and facilitate transfer to long term elderly care.

II. Geriatric Consultation Team Referral Criteria: Age above 65 years having

i. Problems with memory, 
ii. History of 3 unintentional falls in last 6 months,

iii. More than four acute presentations to ED in the last six months,

iv. Multiple health problems or more than five regular medications,

v. Patient or caregiver reports recent functional or behavioral change,

vi. no family or care caregiver.

\section{Investment appraisal}

Focusing on the special care needs of older adults by targeting evaluation on geriatric conditions and improving care transitions, geriatric consultation team can provide an effective environment that enhances the elderly experience and investing high amount of cost in this proposal will balance with improved outcome for older people. Focused assessment and identification of risk $[13,14]$, in order to avoid admission/discharge home. Case management can collaborate and navigate with health/social service agencies to link patients/families with special services, equipment and referrals. Follow up phone support will do by response team (rapid and outreach) deployed to reassess and manage complex cases. Act as a liaison between home, long term care and hospital as required (e.g. regarding system navigation, coordination discharge, and older adult plan of care).

\section{Conclusion}

It is vital that any elderly patient arriving in ED receives immediate assessment by Geriatric consultation team (GCT) by using the bespoken comprehensive geriatric assessment and the appropriate treatment and discharge/transfer to appropriate place is initiated at the earliest opportunity. This emphasizes the role of the GCT as crucial in ensuring that the elderly receive targeted assessment and treatment from specially trained staff in Geriatric Medicine and Care of the Elderly, similar to the model of Pediatric Care in Pediatric Emergency.

\section{References}

1. McKevitt E C, Calvert E, Ng A, Simons R K, Kirkpatrick AW, et al. (2003)
Geriatric trauma: resource use and patient outcomes. Canadian Journal of Surgery. Journal Canadien De Chirurgie, 46(3): 211-215.

2. Hastings S N, Heflin M T (2005) A Systematic Review of Interventions to Improve Outcomes for Elders Discharged from the Emergency Department. Academic Emergency Medicine: Official Journal of the Society for Academic Emergency Medicine 12(10): 978-986.

3. Bratton R L, Short T M (2001) Patient Satisfaction with Telemedicine: A Comparison Study of Geriatric Patients. Journal of Telemedicine and Telecare 7 (2): 85-86.

4. Caplan G A, Williams A J, Daly B, Abraham K (2004) A Randomized, Controlled Trial of Comprehensive Geriatric Assessment and Multidisciplinary Intervention After Discharge of Elderly from the Emergency Department--the Deed Ii Study. Journal of the American Geriatrics Society 52(9): 1417-1423.

5. Carpenter CR, Platts-Mills TF (2013) Evolving prehospital, emergency department, and "inpatient" management models for geriatric emergencies. Clin Geriatr Med 29(1): 31- 47.

6. Hwang U, Morrison R S (2007) The Geriatric Emergency Department: THE GERIATRIC EMERGENCY DEPARTMENT. Journal of the American Geriatrics Society 55(11): 1873-1876.

7. Cimasi R J (2013) Accountable care organizations: value metrics and capital formation. Boca Raton, FL: CRC Press.

8. McClellan M, McKethan A N, Lewis J L, Roski J, Fisher E S (2010) A National Strategy To Put Accountable Care Into Practice. Health Affairs 29(5): 982-990.

9. Jayadevappa R (2012) Quality of Emergency Department Care for Elderly. Emergency Medicine: Open Access 01: e107.

10. Carpenter C R, Heard K, Wilber S, Ginde A A, Stiffler K, et al. (2011) Research Priorities for High-Quality Geriatric Emergency Care: Medication Management, Screening, and Prevention and Functional Assessment: Research Priorities for High-Quality Geriatric Emergency Care. Academic Emergency Medicine 18(6): 644-654.

11. Wilde Larsson B, Larsson G (2002) Development of a short form of the Quality from the Patient's Perspective (QPP) questionnaire. Journal of Clinical Nursing 11(5): 681-687.

12. Gold S, Bergman H (1997) A Geriatric Consultation Team in the Emergency Department. Journal of the American Geriatrics Society 45(6): 764-767.

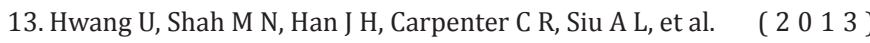
Transforming Emergency Care For Older Adults. Health Affairs 32(12): 2116-2121.

14. Langley G J (2009) The Improvement Guide: A Practical Approach to Enhancing Organizational Performance (2nd ed). San Francisco:

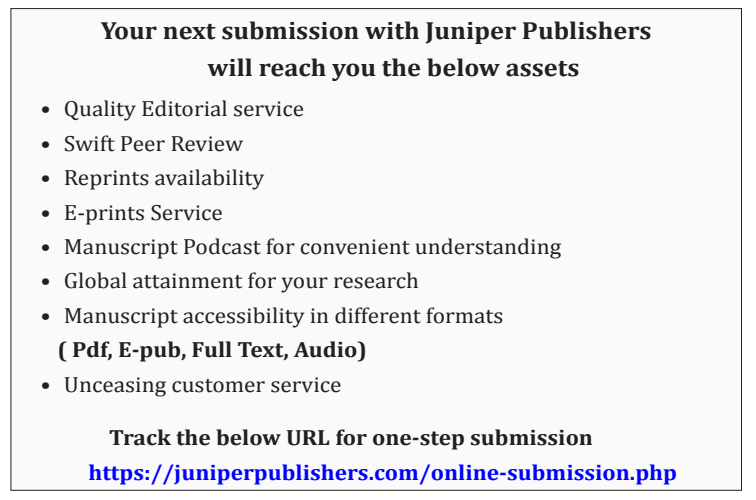

